\title{
Refractive Nonlinearity of Linear Symmetric Molecules and Polymers Revisited
}

\author{
George I. Stegeman ${ }^{* 1,2}$ and Honghua $\mathrm{Hu}^{1}$ \\ ${ }^{1}$ College of Optics and Photonics, Center for Research in Optics and Lasers, University of Central Florida, \\ 4000 Central Florida Blvd, Orlando FL 32816, Florida, USA \\ ${ }^{2}$ Faculty of Physics, Warsaw University of Technology, Koszykowa 75, 00-662 Warszawa
}

Received December 4, 2009; accepted December 11, 2009; published December 31, 2009

\begin{abstract}
In the paper a simple formula for the non-resonant and off-resonant nonlinearity in symmetric linear molecules and conjugated polymers made from such molecules is presented. Obtained results agree with experimental data known from the literature.
\end{abstract}

It is well-known that nonlinear refraction and absorption occur due to transitions between discrete states in molecules and lead to intensity-dependent refractive index and absorption changes $\Delta n=n_{2} I$ and $\Delta \alpha=\alpha_{2} I$ respectively where $I$ is the intensity and $n_{2}$ and $\alpha_{2}$ are coefficients that depend on the detailed structure of the molecules and their environment. The early third harmonic generation experiments by Sauteret and coworkers with input beams at 1.89 and $2.62 \mu \mathrm{m}$ reported the nonlinearities of a number of crystalline conjugated polymers, including $n_{2}=1.8 \times 10^{-12} \mathrm{~cm}^{2} / \mathrm{W}$ in the single crystal conjugated polydiacetylene PTS (poly bis(ptoluene sulfonate) of 2,4-hexadiyne-1,6-diol) [2]. Such large nonlinearities are a consequence of the delocalization of the $2 \mathrm{p}_{\mathrm{z}}$ atomic orbitals of carbon atoms into new molecular $\pi$-orbitals when they are bonded together in linear chains containing single, double or triple bonds. Based on the range of nonlinearities known at that time, these results stimulated both theoretical and experimental interest in linear molecules and conjugated polymers [for example 3-12].

Symmetric linear molecules or polymers have no permanent dipole moment and the electronic states exhibit either even $\left(A_{g}\right)$ or odd $\left(B_{u}\right)$ symmetry. The minimum number of excited states needed to describe their nonlinear optics via electric dipole transitions is two; one state at an energy $\hbar \omega_{10}$ above the ground state is one photon active $\left(1 \mathrm{~B}_{\mathrm{u}}\right)$ and the other at $\hbar \omega_{20}$ is two photon active (and according to convention the state is called $\mathrm{mA}_{\mathrm{g}}$ ) [3]. The ground state is $1 \mathrm{~A}_{\mathrm{g}}$. The states are coupled via transition dipole moment elements $\left|\bar{\mu}_{10}\right|^{2}$ for

*E-mail: george@creol.ucf.edu
$1 \mathrm{~A}_{\mathrm{g}} \rightarrow 1 \mathrm{~B}_{\mathrm{u}}$ and $\left|\bar{\mu}_{21}\right|^{2}$ for $1 \mathrm{~B}_{\mathrm{u}} \rightarrow \mathrm{mA}_{\mathrm{g}}$. It has been shown that that the dominant contributions to the third order nonlinearity in conjugated polymers and "long" linear molecules in general arise from these two excited states, especially for frequencies below the corresponding one and two photon optical resonances at $\bar{\omega}_{10}$ and $\bar{\omega}_{20} / 2$ respectively.[3, 4]

This focused interest led to many measurements of optical nonlinearity linear, symmetric molecules ranging from dilute solutions of the molecules in appropriate solvents, to randomly oriented conjugated polymers in films to single crystal polymers[5-12]. Single crystals or highly oriented films are preferred for applications since the linear chains are parallel to one another which results in an optimally large nonlinearity along the delocalization (chain) axis. Although many measurements of the large nonlinearities were reported near the strongest one photon and two photon resonances of many symmetric linear molecules and conjugated polymers, there are only a few measurements at longer wavelengths beyond these resonances where applications relying on non-resonant nonlinearities and low losses could occur. Here we consider two specific cases, the single crystal polydiacetylene PTS and the linear squairaine molecules. This work was stimulated by independent measurements in PTS from two groups which are at variance with one another, albeit for crystals prepared in very different ways [14-16]. In the squaraines, measurements by different groups are consistent with one another [4, 11, 12 ]. Our goal here is to derive analytical expressions for $n_{2}$ valid in the off-resonance and non-resonant regimes in order to predict at least the sign of the off resonance and nonresonant nonlinearity which in PTS is in dispute.

It is now well known that the molecular third order nonlinearity $\gamma^{(3)}$ for a symmetric linear molecule, corrected for all divergences can be written in the most general case as [18] 


$$
\begin{aligned}
& \gamma_{i j k l}^{(3)}\left(-\left[\omega_{p}+\omega_{q}+\omega_{r}\right] ; \omega_{p}, \omega_{q}, \omega_{r}\right)=\frac{1}{\varepsilon_{0} \hbar^{3}}\left[\sum _ { v , n , m } ^ { \prime } x \left\{\frac{\bar{\mu}_{g v, i} \bar{\mu}_{v n, l} \bar{\mu}_{n m, k} \bar{\mu}_{m g, j}}{\left(\bar{\omega}_{v g}-\omega_{p}-\omega_{q}-\omega_{r}\right)\left(\bar{\omega}_{n g}-\omega_{q}-\omega_{p}\right)\left(\bar{\omega}_{m g}-\omega_{p}\right)}+\right.\right. \\
& +\frac{\bar{\mu}_{g v, j} \bar{\mu}_{v n, k} \bar{\mu}_{n m, i} \bar{\mu}_{m g, l}}{\left(\bar{\omega}_{v g}^{*}+\omega_{p}\right)\left(\bar{\omega}_{n g}^{*}+\omega_{q}+\omega_{p}\right)\left(\bar{\omega}_{m g}-\omega_{r}\right)}+\frac{\bar{\mu}_{g v, l} \bar{\mu}_{v n, i} \bar{\mu}_{n m, k} \bar{\mu}_{m g, j}}{\left(\bar{\omega}_{v g}^{*}+\omega_{r}\right)\left(\bar{\omega}_{n g}-\omega_{q}-\omega_{p}\right)\left(\bar{\omega}_{m g}-\omega_{p}\right)} \\
& \left.+\frac{\bar{\mu}_{g v, j} \bar{\mu}_{v n, k} \bar{\mu}_{n m, l} \bar{\mu}_{m g, i}}{\left(\bar{\omega}_{v g}^{*}+\omega_{p}\right)\left(\bar{\omega}_{n g}^{*}+\omega_{q}+\omega_{p}\right)\left(\bar{\omega}_{m g}^{*}+\omega_{p}+\omega_{q}+\omega_{r}\right)}\right\}-\sum_{n, m}^{\prime}\left\{\frac{\bar{\mu}_{g n, i} \bar{\mu}_{n g, l} \bar{\mu}_{g m, k} \bar{\mu}_{m g, j}}{\left(\bar{\omega}_{n g}-\omega_{p}-\omega_{q}-\omega_{r}\right)\left(\bar{\omega}_{n g}-\omega_{r}\right)\left(\bar{\omega}_{m g}-\omega_{p}\right)}\right. \\
& \left.+\frac{\bar{\mu}_{g n, l} \bar{\mu}_{n g, i} \bar{\mu}_{g m, j} \bar{\mu}_{m g, k}}{\left(\bar{\mu}_{g n, i}^{*} \bar{\mu}_{n g, l} \bar{\mu}_{g m, k} \bar{\mu}_{m g, j}+\omega_{q}\right)\left(\bar{\omega}_{n g}-\omega_{r}\right)\left(\bar{\omega}_{m g}-\omega_{p}\right)}+\frac{\bar{\mu}_{g n, l} \bar{\mu}_{n g, i} \bar{\mu}_{g m, j} \bar{\mu}_{m g, k}}{\left(\bar{\omega}_{n g}^{*}+\omega_{r}\right)\left(\bar{\omega}_{m g}^{*}+\omega_{p}\right)\left(\bar{\omega}_{m g}-\omega_{q}\right)}+\frac{\left(\bar{\omega}_{n g}^{*}+\omega_{r}\right)\left(\bar{\omega}_{m g}^{*}+\omega_{p}\right)\left(\bar{\omega}_{n g}^{*}+\omega_{p}+\omega_{q}+\omega_{r}\right)}{\left(\bar{\omega}_{m}\right)}\right]
\end{aligned}
$$

In symmetric molecules, the largest transition dipole moments involve the ground state and one even and one odd symmetry excited states. Hence the summations over the excited states are over $v, n, m=1\left(1 \mathrm{~B}_{\mathrm{u}}\right)$ and $v, n, m=2\left(\mathrm{~mA}_{\mathrm{g}}\right)$. In condensed matter, the macroscopic third order nonlinearity, including a local field correction $f^{(3)}$, is given by

$$
\begin{aligned}
& \chi_{i j k l}^{(3)}\left(-\left[\omega_{p}+\omega_{q}+\omega_{r}\right] ; \omega_{p}, \omega_{q}, \omega_{r}\right)=N f^{(3)} \bar{\gamma}_{i j k l}^{(3)}\left(-\left[\omega_{p}+\omega_{q}+\omega_{r}\right] ; \omega_{p}, \omega_{q}, \omega_{r}\right) \\
& f^{(3)}=\left[\frac{\varepsilon_{i}^{r}\left(\omega_{p}+\omega_{q}+\omega_{r}\right)+2}{3}\right]\left[\frac{\varepsilon_{j}^{r}\left(\omega_{p}\right)+2}{3}\right]\left[\frac{\varepsilon_{k}^{r}\left(\omega_{q}\right)+2}{3}\right]\left[\frac{\varepsilon_{l}^{r}\left(\omega_{r}\right)+2}{3}\right]
\end{aligned}
$$

in which $N$ is the number of molecules per unit volume. Finally, the electronic nonlinear refractive index coefficient $n_{2}$ is then defined as

$$
n_{2, \mathrm{el}}=\frac{1}{4 n_{x}^{2} \varepsilon_{0} c}\left\{\chi_{\Re, x x x x}^{(3)}(-\omega ; \omega,-\omega, \omega)+\chi_{\Re, x x x x}^{(3)}(-\omega ; \omega, \omega,-\omega)+\chi_{\Re, x x x x}^{(3)}(-\omega ;-\omega, \omega, \omega)\right\},
$$

where the subscript $\mathfrak{R}$ refers to the real part of $\chi^{(3)}$.

Equation (1) contains many terms in the most general case. Kuzyk and Dirk have reduced it considerably for symmetric linear molecules [19]. We have obtained an analytical solution for the "off-resonance" regime, when the lifetimes of the states $\left(\tau_{10}\right.$ and $\left.\tau_{21}\right)$ are neglected in the resonant denominators, i.e. $\left[\bar{\omega}_{10}-\omega\right]>>\bar{\tau}_{10}^{-1}$ and $\left[\bar{\omega}_{10}-2 \omega\right]>>\bar{\tau}_{21}^{-1}$. The expression obtained for linear symmetric molecules is

$$
n_{2}=\frac{N f^{(3)}\left|\bar{\mu}_{10}\right|^{2}}{n_{x}^{2} \varepsilon_{0}^{2} c \hbar^{3}\left(\bar{\omega}_{10}^{2}-\omega^{2}\right)^{2}}\left\{\left|\bar{\mu}_{21}\right|^{2}\left[\frac{\bar{\omega}_{20}\left(\bar{\omega}_{10}^{2}+\omega^{2}\right)+4 \bar{\omega}_{10} \omega^{2}}{\left(\bar{\omega}_{20}^{2}-4 \omega^{2}\right)}+2 \frac{\bar{\omega}_{10}^{2}}{\bar{\omega}_{20}}\right]-\left|\bar{\mu}_{10}\right|^{2}\left[\frac{\bar{\omega}_{10}}{\left(\bar{\omega}_{10}^{2}-\omega^{2}\right)}\left(3 \bar{\omega}_{10}^{2}+\omega^{2}\right)\right]\right\} .
$$

This equation describes the long wavelength (small frequency) "tails" of the dispersion in the nonlinearity. For the "non-resonant" $(\omega \rightarrow 0)$ case

$$
n_{2}=3 \frac{N f^{(3)}\left|\bar{\mu}_{10}\right|^{2}}{n_{x}^{2} \varepsilon_{0}^{2} c \hbar^{3} \bar{\omega}_{10}^{3}}\left\{\frac{\bar{\omega}_{10}}{\bar{\omega}_{20}}\left|\bar{\mu}_{21}\right|^{2}-\left|\bar{\mu}_{10}\right|^{2}\right\} \text {. }
$$

Note that that the interference between the one and two photon contributions proportional to $\left(\left|\bar{\mu}_{10}\right|^{2}\right)$ and $\left(\left|\bar{\mu}_{21}\right|^{2}\right)$ respectively can be actually detrimental to obtaining large non-resonant nonlinearities! Another important factor is the ratio of the energies of the two states above the ground state. Furthermore, since the two terms have both different signs and different frequency dispersion, the net sign of $n_{2}$ can depend strongly on frequency. To support the conclusions from the equations, we have evaluated Eqn. (4) numerically under no approximations. The result is illustrated in Figs. 1 and 2 which show the frequency dispersion of $n_{2}$ typical of polydiacetylenes and squaraines. The ratios of the transition dipole moments were taken from the theoretical work of Soos and co-workers and Kuzyk and co-workers respectively $[3,4]$ Note that as predicted by Eqn. (4), the sign of the nonlinearity can change with photon frequency depending on the relative magnitude of $\bar{\omega}_{10}\left|\bar{\mu}_{21}\right|^{2} / \bar{\omega}_{20}\left|\bar{\mu}_{10}\right|^{2}$. For both the polydiacetylenes and squaraines the sign of $n_{2}$ is initially dominated by the one photon resonance, is always negative and increasing as the optical frequency is reduced below the one photon resonance. As the two photon resonance is approached, interference occurs between the one and two photon resonant contributions. $n_{2}$ in the frequency range approaching the two photon resonances, on two photon resonance and for longer wavelengths the sign depends on the values of $\left|\bar{\omega}_{10}-\omega\right| \bar{\tau}_{10}$ and $\left|\bar{\omega}_{20}-\omega\right| \bar{\tau}_{21}$, and on the ratio $\bar{\omega}_{10}\left|\bar{\mu}_{21}\right|^{2} / \bar{\omega}_{20}\left|\bar{\mu}_{10}\right|^{2}$. The dispersion in $n_{2}$ resembles that associated with the dispersion in the linear index, but is opposite in sign. It becomes more pronounced when the lifetimes of the states increases as shown in Figs. 1b and 1c. If the ratio $\bar{\omega}_{10}\left|\bar{\mu}_{21}\right|^{2} / \bar{\omega}_{20}\left|\bar{\mu}_{10}\right|^{2}$ is greater than unity the sign of the non-resonant $n_{2}$ is positive, and for ratios less than one the sign of the non-resonant $n_{2}$ is negative. In fact, the nonlinearity predicted for the polydiacetylene PTS based on the measured and calculated transition dipole moments $\left(4>\left|\bar{\mu}_{21}\right|^{2} /\left|\bar{\mu}_{10}\right|^{2}>2\right)$ and excited state lifetimes $\left(\bar{\tau}_{10} \approx\right.$ few ps, $\left.\bar{\tau}_{21} \approx 100 \mathrm{fs}\right)$ is shown in Fig $1(\mathrm{c})$. Experiments at CREOL single crystal PTS agree with the above 


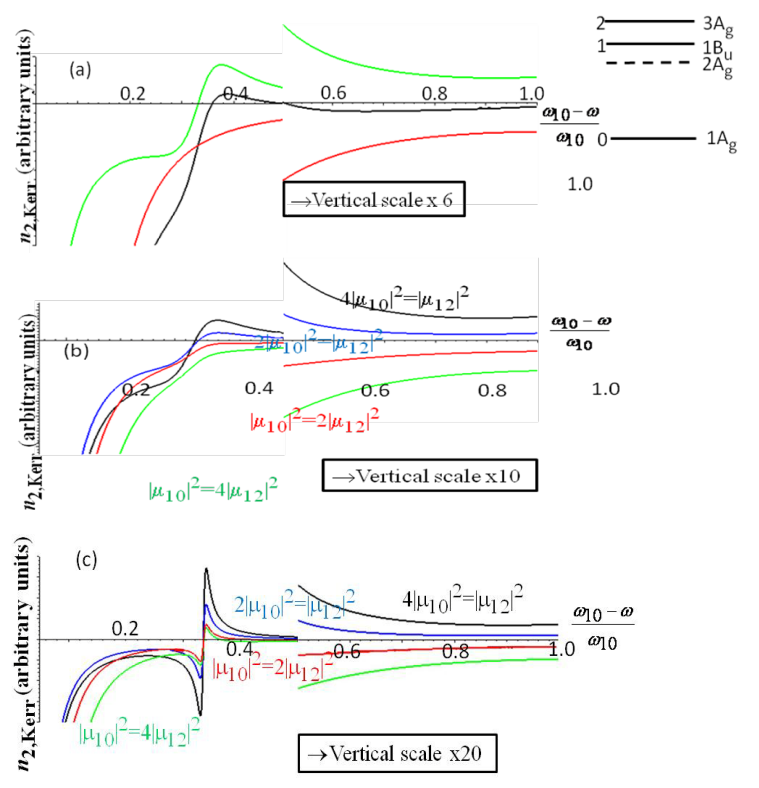

Fig. 1. Frequency dispersion of $n_{2, \text { Kerr }}$ for $\bar{\Gamma}_{21}=10 \bar{\Gamma}_{10}, \omega_{20}=1.33 \bar{\omega}_{10}$ and different relative values of $\left|\bar{\mu}_{12}\right|^{2} /\left|\bar{\mu}_{10}\right|^{2}$. (a) For $\left|\bar{\mu}_{10}\right|^{2}=\left|\bar{\mu}_{21}\right|^{2}$, the contribution due to $\left|\bar{\mu}_{10}\right|^{2}$ (red line), $\left|\bar{\mu}_{21}\right|^{2}$ (green line) and the sum of the two (black line) are shown. (b) The sum of the contributions for different relative $\bar{\Gamma}_{10} / \omega_{10}=0.01$ values of the transition dipole moments. $\bar{\Gamma}_{10} / \omega_{10}=0.01$ in (a) and (b). (c) $\bar{\Gamma}_{10} / \omega_{10}=0.001$. Typical parameters for polydiacetylenes were used.

positive on the low frequency side has been observed, as well as the decay in the magnitude of $n_{2}$ for long wavelengths $[16,19]$. Similar behavior has been reported in conjugated phenylenevinylenes another symmetric linear polymer with $\pi$-electron delocalization. Specifically Bubeck and co-workers actually tracked the sign change in $n_{2}$ through the zero crossing point in $\mathrm{MEH}$ polyphenylvinylene [10]. In both materials, the sign of $n_{2}$ is negative between the one and two photon resonances, becomes positive near the two photon resonance and generally remains positive towards zero frequency (nonresonant case).

In contrast to this behavior, the nonlinearity in linear, symmetric molecules called squaraines have weaker two photon transition strengths and therefore should remain negative over the full range $\bar{\omega}_{10}>\omega>0$. Specific predictions are shown in Fig. 2. In that class of materials the ratio $\bar{\omega}_{10}\left|\bar{\mu}_{21}\right|^{2} / \bar{\omega}_{20}\left|\bar{\mu}_{10}\right|^{2}$ is significantly smaller than unity because $\left|\bar{\mu}_{21}\right|^{2}<\left.\bar{\mu}_{10}\right|^{2}$. Experimental measurements support a negative near-resonant nonlinearity [4, 11,12].

Very recently the non-resonant nonlinearity has been measured in the symmetric linear molecule $\mathrm{CS}_{2}$. The transition dipole moments were measured to be $\left|\bar{\mu}_{21}\right|^{2}>\left|2 \bar{\mu}_{10}\right|^{2}$ by two photon absorption spectroscopy and the nonlinearity $n_{2}$ was found to be positive, as predicted above [21].

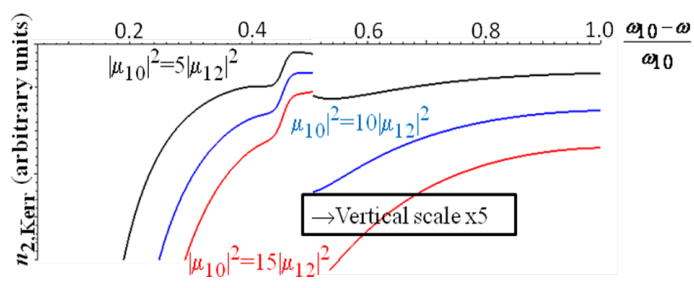

Fig. 1. Frequency dispersion of $n_{2, \text { Kerr }}$ for $\bar{\Gamma}_{21}=1.3 \bar{\Gamma}_{10}, \omega_{20}=1.10 \bar{\omega}_{10}$ and different relative values of $\left|\bar{\mu}_{12}\right|^{2} /\left|\bar{\mu}_{10}\right|^{2}$. Parameters used are typical of squaraines.

In summary, we have obtained a simple formula for the non-resonant and off-resonant nonlinearity in symmetric linear molecules and conjugated polymers made from such molecules. The sign of the non-resonant nonlinearity depends critically on the ratio of molecular parameters, specifically on $\bar{\omega}_{10}\left|\bar{\mu}_{21}\right|^{2} / \bar{\omega}_{20}\left|\bar{\mu}_{10}\right|^{2}$ and the excited state lifetimes. The results should be useful in predicting not only the sign, but also the magnitude of the nonlinearity, within the approximations associated with local field.

\section{References}

[1] Y. R. Shen, The Principles of Nonlinear Optics, Wiley Intersciences series ( J. Wiley and Sons, New York, 1984); R. W. Boyd, Nonlinear Optics, (Academic Press, San Diego, 1992

[2] C. Sauteret, J.P. Hermann, R. Frey, F. Pradere, J. Ducuing, R.H. Baughman, R.R. Chance, Phys. Rev. Lett., 36, 956 (1976).

[3] Z. G. Soos, P. C. M. McWilliams and G. W. Hayden, Chem. Phys. Lett., 171, 14-18 (1990); P. C. M. McWilliams, G. W. Hayden and Z. G. Soos, Phys. Rev. B, 43, 9777-9791 (1991)

[4] K. S. Mathis, M. G. Kuzyk, C. W. Dirk, A. Tan, S. Martinez and G. Gampos, J. Opt. Soc. Am. B, 15, 871-883 (1998)

[5] G. M. Carter, Y. J. Chen and S. K. Tripathy, Opt. Eng., 24, 609 (1985)

[6] J. Bolger, T. G. Harvey, W. Ji, A. K. Kar, S. Molyneux, B.S. Wherrett, D. Bloor, P. Norman, J. Opt. Soc. Am. B, 9, 1552 (1992)

[7] P. A. Gass, I. Abram, R. Raj, and M. Schott, J. Chem. Phys., 100, 8897 (1994)

[8] T. Kobayashi, Synth. Metals, 49-50, 565-581 (1992)

[9] M. Samoc, A. Samoc and B. Luther-Davies, Synth. Met., 109, 79-83 (2000)

[10] reviewed in A. Bahtiar, K. Koynov, A. Kibrom, T. Ahn, and Chr. Bubeck, Proc. of SPIE, 6330, 63300C-14 (2006)]

[11] D. W. Garvey, Q. Li, M. G. G. Kuzyk, C. W. Dirk and S. Martinez, Opt. Lett., 21, 104-106 (1996)

[12] S.-J. Chung et al, J. Am. Chem. Soc., 128, 14444-14445 (2006)

[13] B. Lawrence, W. Torruellas, M. Cha, G. I. Stegeman, J. Meth, S. Etemad, and G. Baker, Phys. Rev. Lett. 73, 597 (1994)

[14] B. L. Lawrence, M. Cha, W. E. Torruellas, G. I. Stegeman, S. Etemad and G. Baker, Appl. Phys. Lett., 64, 2773 (1994)

[15] R. Quintero-Torres and M. Thakur, J. Appl. Phy., 85, 401-3 (1999)

[16] S. Polyakov, F. Yoshino, M. Liu, and G. Stegeman, Phys. Rev. B 69, 115421-11 (2004)]

[17] M. Samoc, A. Samoc, B. Luther-Davies and M. Woodruff, Pure and Applied Optics, 5, 681-7 (1996)

[18] B. J. Orr and J. F. Ward, Mol. Phys., 20, 513-526 (1971)

[19] M. G. Kuzyk and C. W. Dirk, Phys. Rev. A, 41, 5098-5109 (1990).

[20] W. E. Torruellas, B. L. Lawrence, G. I. Stegeman and G. Baker, Opt. Lett., 21,1777-9 (1996)

[21] H. Hu, S. Webster, D. Hagan and E. W. Van Stryland, to be published

[22] C. W. Dirk, L.-T. Cheng and M. G. Kuzyk, Int. J. Quant. Chem., 43, 27-36 (1992) 